\title{
RELATIONS ENTRE LA TOPOGRAPHIE AQUATIQUE ET L'ORGANISATION SPATIALE DE L'ICHTYOFAUNE LACUSTRE : DÉFINITION DES MODALITÉS SPATIALES D'UNE STRATÉGIE DE PRÉLĖVEMENT REPRODUCTIBLE (*)
}

\author{
F. DEGIORGI (1) et J.P. GRANDMOTTET (2) \\ avec la collaboration technique de \\ Ph. Chanteloube (1), C. PARdon (1), A. ROUSSElet (3), \\ J.F. SUAT (1) et J.Ph. VANDELLE (1)
}

(1) Laboratoire d'Hydrobiologie-Hydroécologie, Institut des Sciences et Techniques de l'Environnement, Université de Franche-Comté,place Leclerc, 25030 Besançon Cedex, France.

(2) Maison Nationale de L'Eau et de la Pêche, 25290 Ornans, France.

(3) Garde-pêche commissionné du Conseil Supérieur de la Pêche (détaché auprès de la Fédération Départementale de Pêche du Doubs, France).

\section{RÉSUMÉ}

Afin d'étudier l'organisation spatio-temporelle de l'ichtyofaune de systèmes lacustres homogènes, plusieurs campagnes saisonnières de pêche ont été réalisées sur deux lacs du massif jurassien en utilisant des filets verticaux à enrouleur. Ce dispositif, qui prospecte toute la tranche d'eau quel que soit le site où il est installé, permet en effet d'élaborer une stratégie d'échantillonnage rigoureuse en divisant a priori le plan lacustre autour de descripteurs topographiques considérés comme des pôles exerçant une attraction différentielle sur les poissons. Durant chaque campagne, la prospection simultanée de l'ensemble des pôles d'attraction identifiés a été répétée six fois.

Des analyses factorielles des correspondances globales, puis inter et intra-groupes, ont alors été effectuées d'une part sur chaque jeu saisonnier de captures par unité d'effort groupées par pôles pour évaluer les variabilités spatiales, et d'autre part par séquences de prospection simultanée de l'ensemble des pôles pour analyser les variabilités temporelles.

Cette approche fait apparaître à la fois une organisation logique nette des espèces autour des postes prospectés ainsi que le caractère dynamique de l'attractivité des pôles. La stabilité des images globales successives obtenues par la répétition des séquences de prospection simultanée de l'ensemble des compartiments montre la pertinence de la partition réalisée.

( $\left.{ }^{\star}\right)$ Recherches faisant partie d'un programme soutenu par le Conseil Supérieur de la Pêche. 


\title{
SPATIAL RELATIONS BETWEEN LACUSTRINE ICHTYOFAUNA AND A PRE-DEFINED PARTITION OF THE LACUSTRINE BASIN
}

\begin{abstract}
In order to study the spatial organization of the ichtyofauna of homogeneous lakes, several seasonal fishing campaigns have been carried out in two lakes of the Jura using vertical roller gill nets. This technique, which prospects the entire depth of water wherever it is disposed, allows to make a rigourous sampling strategy by dividing the plan of the lake according to pre-defined topogaphic descriptors considered as poles which creat a differential attraction on the different species of fishes. For each sampling campaign, the simultaneous prospection of all the different attraction poles have been repeated six times.
\end{abstract}

Global, then inter and intra-group correspondence analysis have been made on every seasonal series of catches per unit effort grouped by pole in order to estimate spatial variability, and by sampling sequences in order to analyse the temporal variability.

This approach shows a strong logic organization of species around the posts prospected and the dynamic aspect of the attraction of the pre-defined poles.

\section{INTRODUCTION}

Dans le cas des systèmes lacustres, les relations entre les poissons et les composantes physiques de leur environnement sont souvent étudiées à l'aide de descripteurs caractérisant à chaque fois un lac tout entier, afin de déterminer les associations d'espèces inféodées à des "niches" (LEGENDRE et BEAUVAIS, 1978) ou d'élaborer une typologie des peuplements pisciaires (TONN et MAGNUSON, 1982). Des approches similaires sont réalisées pour des lacs de grandes dimensions (plusieurs dizaines de $\mathrm{km}^{2}$ ) caractérisés par une zonation marquée ou des gradients induisant une sectorisation géographique nette du plan d'eau (GASCON et LEGGET, 1977 ; BARLA, 1991).

En revanche, dans le cas des systèmes lotiques, l'organisation spatiale des différentes espèces a non seulement été analysée à l'échelle du cours d'eau tout entier (zonation d'HUET, 1949 ou d'ILIES et BOTOSANEANU, 1963 ; typologie longitudinale de VERNEAUX, 1973), mais aussi au sein de tronçons homogènes, afin de déterminer le degré d'affinité des poissons avec différents "habitats" (BOVEE, 1978 ; GORMANN et KARR, 1978 ; MOREAU et LEGENDRE, 1979 ; SAVARD et MOREAU, 1982).

En ce qui concerne les lacs moins hétérogènes, c'est-à-dire ne présentant pas de différences typologiques internes, abritant la plupart du temps un petit nombre d'espèces, les approches réalisées portent essentiellement sur les exigences en matière d'habitat d'une espèce en particulier (DIANA et al., 1977 ; LINDSTRÖM et BERGSTRAND, 1979 ; HINDAR et JONSSON, 1982 ; HARALDSTAD et JONSSON, 1983).

Suivant une démarche différente, notre propos consistera à tenter d'appréhender l'organisation spatiale de l'ensemble des espèces de la communauté piscicole dans le cas de deux lacs du massif jurassien, de dimensions restreintes (78 et 95 ha pour 30 et 29 mètres de profondeur maximum) et dont l'ichtyofaune présente une variété spécifique relativement réduite (6 et 10 espèces). Dans ce contexte, nous nous sommes attachés à étudier les variations spatio-temporelles des combinaisons réalisées par les poissons à travers la mosaïque habitationnelle en analysant, conformément aux recommandations de MOREAU et LEGENDRE (1979), les abondances relatives que constituent les captures par unité d'effort de pêche au filet et non le simple critère "présence-absence".

Cette approche est basée sur un découpage a priori de l'espace lacustre qui nous a permis d'élaborer une stratégie d'échantillonnage précise (FRONTIER, 1983; SHERRER, 1983), puis d'évaluer et d'interpréter la variabilité du modèle proposé. 


\section{MÉTHODE}

\subsection{Caractéristiques des milieux étudiés}
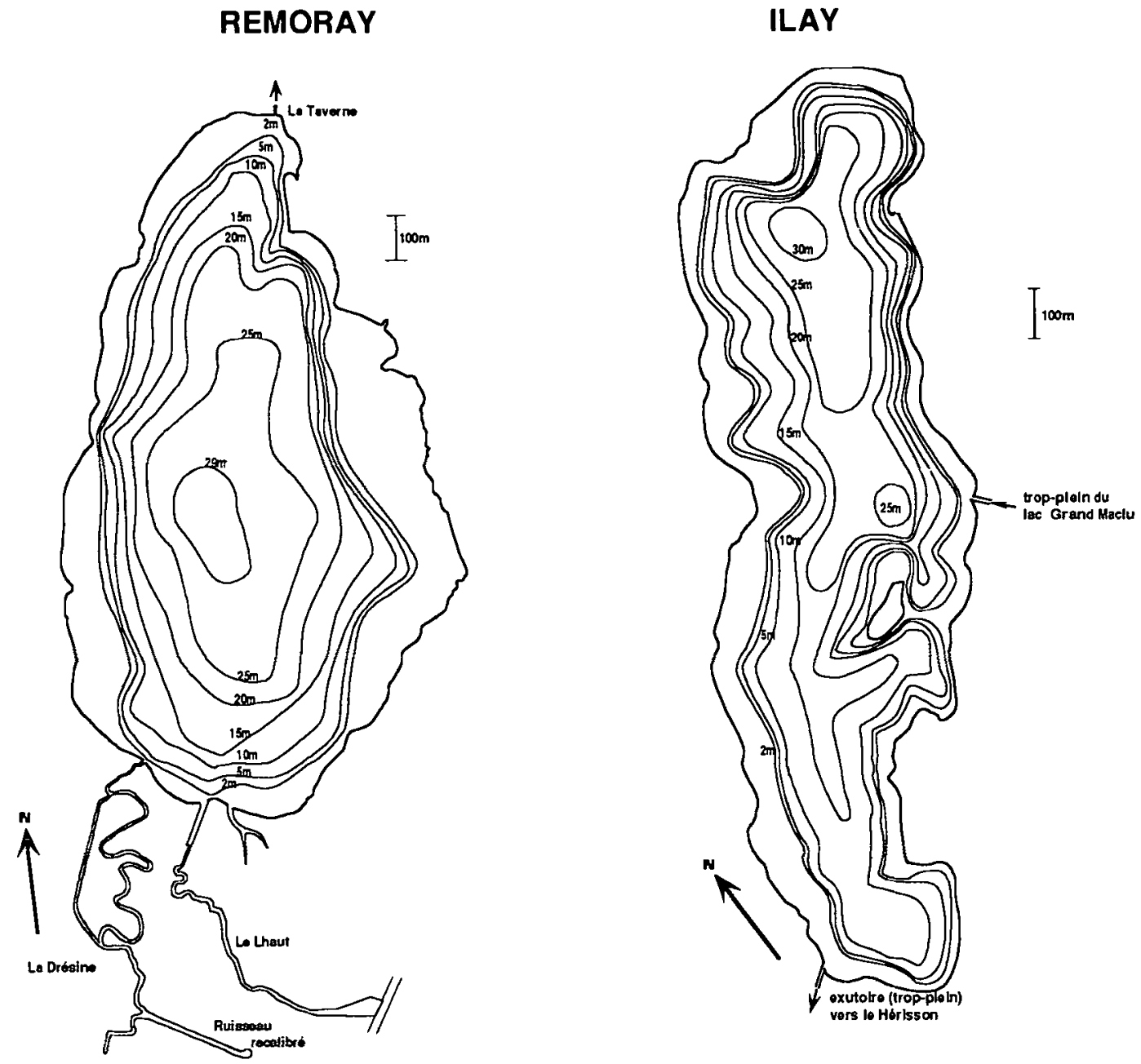

Figure 1 : Bathymétrie des lacs d'llay et de Remoray.

Figure 1 : Bathymetric maps of the llay and Remoray lakes.

Les principales caractéristiques morphologiques des lacs d'ilay et de Remoray sont détaillées dans le tableau I. Si ces deux lacs appartiennent aux mêmes classes de surface et de profondeur, ils diffèrent notoirement par la morphologie de leur cuvette (figure 1) et par la nature de leur alimentation, essentiellement diffuse dans le cas d'llay et assurée majoritairement par deux afférences pour le lac de Remoray. Le métabolisme de ces deux systèmes holo-dimictiques du deuxième ordre thermique (sensu HUTCHINSON, 1957) est caractérisé par une teneur en calcium moyenne pour llay (40 à $55 \mathrm{mg} / \mathrm{l}$ sur 8 mesures) et forte pour Remoray (63 à $75 \mathrm{mg} / \mathrm{l}$ sur 8 mesures), par une désoxygénation estivale non négligeable de l'eau de fond et par une production primaire (biomasse) faible (VERNEAUX et al., 1991) . 


\section{Tableau I : Caractéristiques morphologiques et hydrologiques des lacs d'llay et de Remoray .}

Table I : Morphological and hydrological characteristics of the llay and Remoray lakes.

\begin{tabular}{lcc}
\hline Caractéristiques & ILAY & REMORAY \\
\hline Altitude & $778 \mathrm{~m}$ & $851 \mathrm{~m}$ \\
Superficie & $71,8 \mathrm{ha}$ & $95,0 \mathrm{ha}$ \\
Surface littorale $(z<3 \mathrm{~m})$ & 19,9 ha $(27,7 \%)$ & $29,5 \mathrm{ha}(29,5 \%)$ \\
Surface envégétée & $16,4 \mathrm{ha}(27,7 \%)$ & $9,3 \mathrm{ha}(9,8 \%)$ \\
Profondeur max. & $31 \mathrm{~m}$ & $29 \mathrm{~m}$ \\
Profondeur moy. & $11,5 \mathrm{~m}$ & $10,1 \mathrm{~m}$ \\
Périmètre & $4875 \mathrm{~m}$ & $4635 \mathrm{~m}$ \\
Longueur max. & $1840 \mathrm{~m}$ & $1600 \mathrm{~m}$ \\
Largeur max. & $475 \mathrm{~m}$ & $900 \mathrm{~m}$ \\
\hline Alimentation & trop-plein d'un lac adjacent (karst?) & 2 afférences (karst?) \\
Exutoire & 1 trop-plein +percolations karstiques & 1 efférence \\
B. V. géographique & $5,2 \mathrm{~km}$ 2 (les 2 lacs adjacents inclus) & $27,7 \mathrm{~km}$. \\
\hline
\end{tabular}

\subsection{Protocole d'échantillonnage}

La prospection de l'espace lacustre a été réalisée au moyen de filets verticaux à enrouleur de 2 mètres de large, semblables à ceux décrits par de nombreux auteurs anglosaxons (HARTMANN, 1962 ; HORAK et TANNER, 1964 ; BARTOO et al., 1973) et déjà utilisés sur les lacs jurassiens par GRANDMOTTET et VAUDAUX (1989), GUYARD et al. (1989) ainsi que par DEGIORGI et GRANDMOTTET (1992) qui ont montré les avantages de ce dispositif de capture.

L'unité d'échantillonnage est constituée, pour notre étude, d'un ensemble de six filets verticaux (appelé "batterie") dont le vide de mailles varie de 10 à $60 \mathrm{~mm}$ par pas de $10 \mathrm{~mm}$ et qui sont disposés pendant 24 heures sur le même site (appelé poste) et ce quelle qu'en soit la profondeur puisque ces filets, de hauteur modulable, prospectent à chaque fois l'intégralité de la tranche d'eau. L'homogénéité des engins de pêche, préconisée par RICKER (1980), lorsque l'on se sert de l'analyse des prises par unité d'effort de pêche pour étudier les communautés piscicoles, est ainsi assurée. En outre, cette propriété du dispositif utilisé permet de s'affranchir de la dimension verticale de l'espace lors de l'élaboration de la stratégie d'échantillonnage qui consiste en effet à programmer la disposition des filets sur le plan lacustre, en fonction des différents habitats qui peuvent y être identifiés, sans se préoccuper, a priori, des différentes strates de profondeur.

Afin de gagner en efficacité et de tenir compte de la répartition des populations ciblées, il convenait en effet de choisir de façon non hasardeuse les sites prospectés. Or, des analyses bibliographiques et des investigations antérieures menées par enquête, filets maillants traditionnels et verticaux (GRANDMOTTET, 1983 ; CEEC, 1988 ; GRANDMOTTET et VAUDAUX, 1989 ; GUYARD et al.,1989) nous ont conduit à relativiser la notion d' "habitat" en ce qui concerne l'ichtyofaune lacustre. En effet, nous avons pu. observer d'importantes variations qualitatives et quantitatives de la fréquentation de la plupart des "habitats" d'un lac à l'autre, mais aussi d'une saison à l'autre, et même d'un jour à l'autre selon le stade de développement des individus, les conditions météorologiques et les rythmes nycthéméraux. En revanche, à l'issue de ces investigations préalables, il nous est apparu que cette répartition dynamique des poissons dans l'espace lacustre semblait conditionnée par l'attractivité de différents pôles définis par la topographie aquatique (relief de la cuvette, hauteur d'eau, encombrement spatial des substrats, proximité avec d'autres éléments de la mosaïque,...). Dans ce contexte, nous avons décidé de réaliser un échantillonnage stratifié (FRONTIER, 1983 ; SHERRER, 1983) en découpant l'espace lacustre autour de pôles d'attraction définis a priori, qui seront prospectés méthodiquement et simultanément, de façon à percevoir l'ensemble des mouvements des différentes espèces. 
La stratification de l'échantillonnage a été opérée à deux niveaux. D'abord, l'espace lacustre a été partagé en 3 zones morphostructurales utilisées différemment par les espèces rencontrées en système lacustre (CEEC, 1988). Cette première division, schématisée sur la figure 2 et illustrée par l'exemple de la figure 3, s'appuie sur la structure caractéristique de la cuvette des lacs à morphologie différenciée (HUTCHINSON, 1957 ; DUSSART, 1966) :

- la zone littorale, délimitée par la rupture de pente de la beine qui s'effectue, dans le cas des deux lacs étudiés, comme pour la plupart des lacs naturels du Jura, entre les isobathes -2 et -3 mètres,

- le talus, délimité par les ruptures de pente de la beine (limite supérieure) et de la plaine (limite inférieure),

- la zone centrale, constituée par la masse d'eau située au-dessus de la plaine.
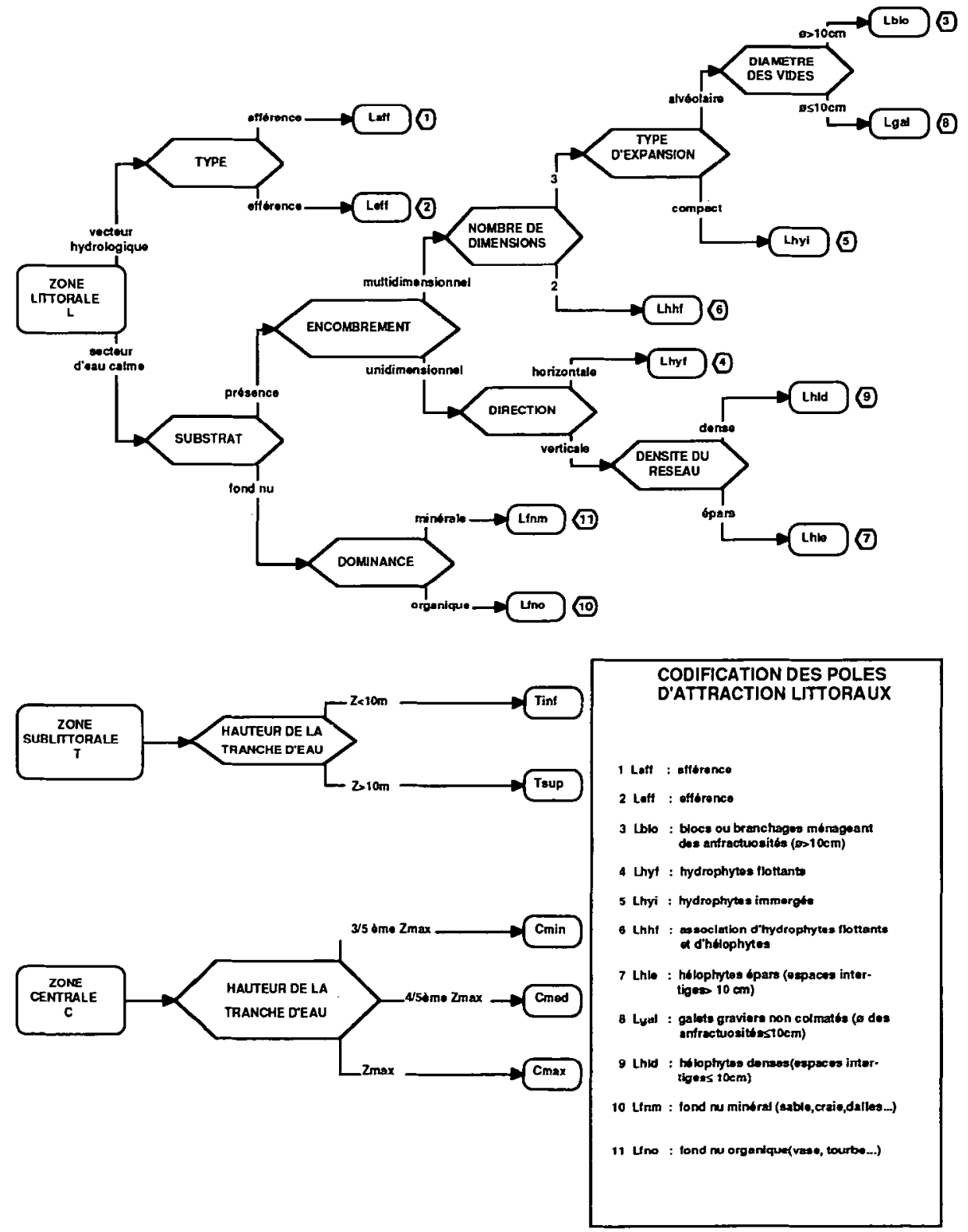

Figure 2 : Schéma directif de description de l'espace lacustre.

Figure 2 : Descriptive and directive division of lacustrine space. 
Parallèlement, une liste des pôles d'attraction existant dans la cuvette lacustre est dressée en se référant au schéma de description de l'espace lacustre présenté à la figure 2. Celui-ci a été établi par regroupement des"habitats-poissons" définis par GRANDMOTTET (1983). Les éléments topographiques servant à la définition des pôles d'attraction se rapportent aux trois variables physiques principalement utilisées pour la définition des habitats aquatiques (MOREAU et LEGENDRE, 1979 ; SAVARD et MOREAU, 1981 ; TONN et MAGNUSON, 1982) :

- la hauteur d'eau constitue un stratificateur variant continûment (FRONTIER, 1983 ; SHERRER, 1983) qui détermine l'ambiance physico-chimique au ras du sédiment (lumière, oxygénation, température, ...) ; une division arbitraire des zones sublittorale et centrale a donc été opérée d'après ce descripteur ;

- les vecteurs hydrologiques (afférences et efférences) déterminent des compartiments possédant des caractéristiques particulières, quel que soit le substrat sousjacent ; en revanche, les courants lacustres, d'orientation inconstante et de localisation diffuse n'ont pas été pris en compte ;

- la structure et l'encombrement des substrats minéraux et des supports végétaux paraissent intervenir davantage que leur nature proprement dite (CROWDER et COOPER, 1982 ; GRANDMOTTET,1983 ; DIEHL,1988 ; DIONNE et FOLT, 1991) : le développement spatial des éléments de la topographie et en particulier la forme et la taille des "vides" ou espaces utilisables par les poissons sont utilisés pour définir les différents pôles.

Dans chaque zone, chaque surface homogène de plus de $200 \mathrm{~m}^{2}$ (encombrement d'une batterie disposée en quinconce) est affectée à un pôle d'attraction suivant le schéma de description directive de la figure 2 ; appelons " $m$ " le nombre de pôles inventoriés pour une campagne donnée.

Pour les deux lacs étudiés, il a ainsi été réalisé, avant chaque campagne, une cartographie des $\mathrm{m}$ pôles d'attraction qui ont été ensuite prospectés de façon répétitive et aléatoire. A titre d'exemple, la cartographie des pôles d'attraction relevés en été sur le lac de Remoray est reproduite sur la figure 3 où sont également localisés les postes échantillonnés. Les lacs d'llay et de Remoray ont été prospectés respectivement en 1989 et 1990/1991 durant 2 et 3 campagnes saisonnières, en évitant pour les différentes espèces en présence, les périodes de reproduction qui amplifient aléatoirement l'activité et donc la vulnérabilité (RICKER, 1980) de l'espèce concernée par la fraie. Durant chaque campagne, les $\mathrm{m}$ pôles d'attraction inventoriés ont été prospectés 6 fois selon le même effort unitaire et sur des postes (localisations) différents (cf. tableau II et figure 3 ).

Sur le plan temporel, les $\mathrm{m}$ pôles sont prospectés simultanément (ou quasi simultanément) de façon à produire le même effort global (appelé aussi "séquence") qui regroupe donc, dans un laps de temps le plus court possible, la prospection unitaire des $\mathrm{m}$ postes affectés respectivement aux $\mathrm{m}$ pôles définis tandis qu'une campagne est constituée de 6 efforts globaux successifs. Le nombre de batteries de 6 filets disposés par campagne s'élève donc à $6 \mathrm{~m}$. 


\section{LEGENDE}

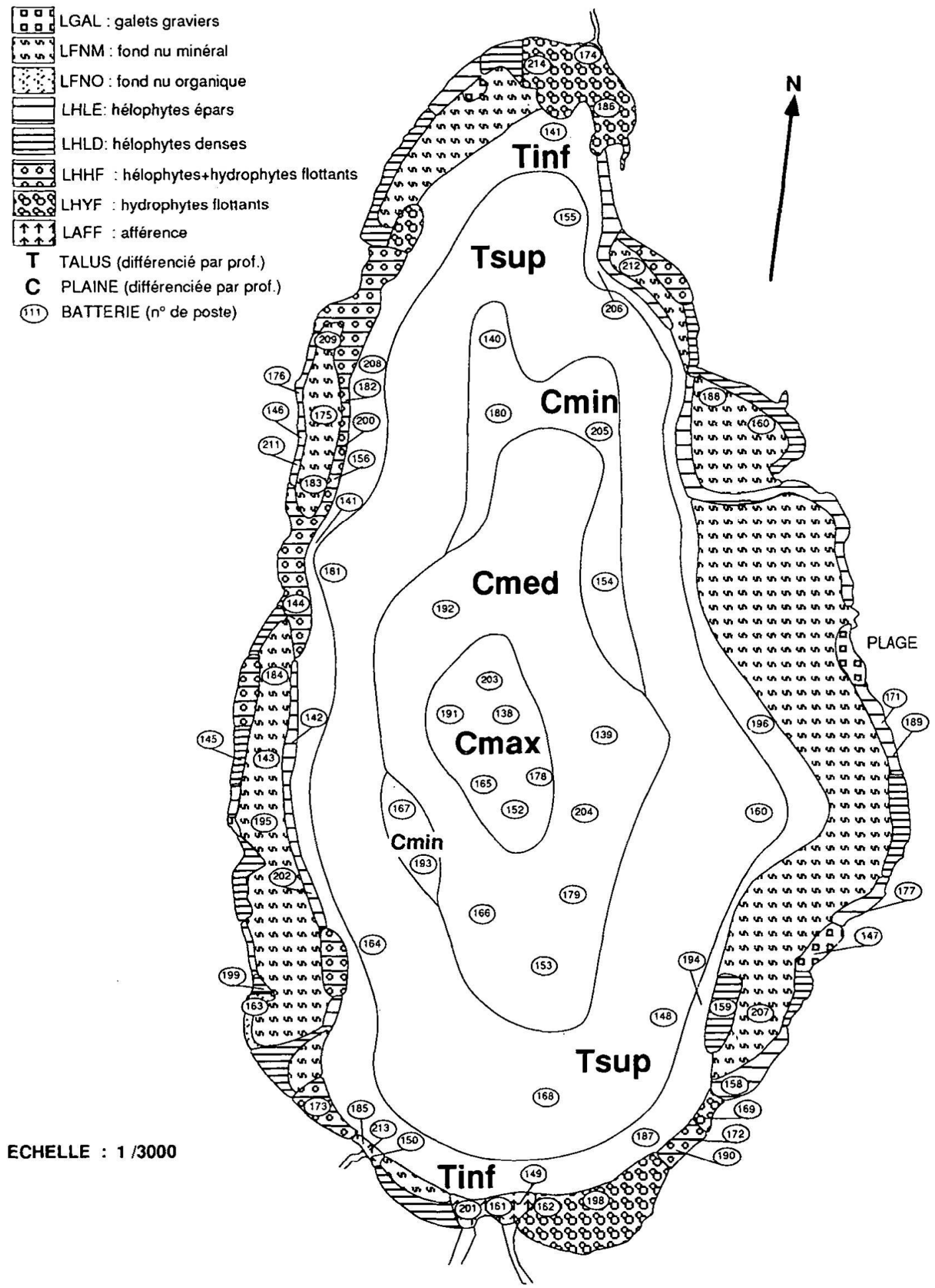

Figure 3 : Cartographie des 11 pôles d'attraction relevés en été sur le lac de Remoray.

Figure 3 : Cartography of the eleven poles prospected in Remoray lake in summer. 
Tableau II : Listes et surfaces relatives des compartiments d'attraction prospectés lors de chacune des 5 campagnes réalisées sur les 2 lacs.

Table II : List and relative area of attraction poles prospected during each of the 5 campaigns carried out in the two lakes.

\begin{tabular}{|c|c|c|c|c|c|}
\hline \multirow{2}{*}{$\begin{array}{c}\text { LAC } \\
\text { CAMPAGNE } \\
\text { PÔLES } \\
\end{array}$} & \multicolumn{2}{|c|}{ ILAY } & \multicolumn{3}{|c|}{ REMORAY } \\
\hline & PRINTEMPS & ETE & ETE & AUTOMNE & PRINTEMPS \\
\hline CMAX & $11,9 \%$ & $11,9 \%$ & $2,8 \%$ & $2,8 \%$ & $2,8 \%$ \\
\hline CMED & $7,1 \%$ & $7,1 \%$ & $13,0 \%$ & $13,0 \%$ & $13,0 \%$ \\
\hline CMIN & $15,4 \%$ & $15,4 \%$ & $4,2 \%$ & $4,2 \%$ & $4,2 \%$ \\
\hline TSUP & $19,3 \%$ & $19,3 \%$ & $23,4 \%$ & $23,4 \%$ & $23,4 \%$ \\
\hline TINF & $17,3 \%$ & $17,4 \%$ & $31,9 \%$ & $31,9 \%$ & $31,9 \%$ \\
\hline LAFF & - & - & $0,8 \%$ & $\hat{0}, 8 \%$ & $0,8 \%$ \\
\hline LBLO & $1,3 \%$ & $1,3 \%$ & - & - & - \\
\hline LEFF & - & - & - & - & $0,8 \%$ \\
\hline LFNM & $5,5 \%$ & $4,9 \%$ & $14,5 \%$ & $15,5 \%$ & $18,8 \%$ \\
\hline LFNO & - & - & $0,0 \%$ & $0,0 \%$ & $0,0 \%$ \\
\hline LGAL & - & - & $0,0 \%$ & $0,0 \%$ & $0,9 \%$ \\
\hline LHLD & $5,4 \%$ & $9,0 \%$ & $2,6 \%$ & $3,9 \%$ & $0,0 \%$ \\
\hline LHLE & $7,6 \%$ & $4,5 \%$ & $2,5 \%$ & $4,5 \%$ & $3,4 \%$ \\
\hline LHHL & $7,4 \%$ & $7,5 \%$ & $2,4 \%$ & $0,0 \%$ & - \\
\hline LHYF & $1,9 \%$ & $1,9 \%$ & $2,0 \%$ & $0,0 \%$ & - \\
\hline $\begin{array}{c}\mathrm{m} \text { : nombre } \\
\text { de pôles pris } \\
\text { en compte }\end{array}$ & 11 & 11 & 11 & 9 & 9 \\
\hline
\end{tabular}

\subsection{Méthodes d'analyse des données}

Les résultats obtenus constituent des matrices d'indices d'abondance numérique dont les colonnes représentent les captures par espèce, tandis que les lignes figurent les prises effectuées sur chaque poste, ceux-ci étant regroupés par pôle et rangés suivant l'ordre chronologique de leur prospection. Ces tableaux sont donc caractérisés par une organisation tridimensionnelle : espace $\mathrm{x}$ temps $\mathrm{x}$ espèces, dont l'intérêt et la spécificité sont soulignés par DAKKI (1985) ou DOLEDEC et CHESSEL $(1987,1989,1991)$.

Pour analyser ces données, nous avons choisi d'utiliser l'Analyse Factorielle des Correspondances ou AFC ( BENZECRI et al., 1973 ; HILL, 1973). En effet, les matrices de données comprennent un grand nombre de zéro. Dans ce cas, qui laisse présupposer des gradients prononcés entre objets, "l'Analyse des Correspondances produit des ordinations plus satisfaisantes que l'analyse en composante principale" (HILL, 1973 ; GAUCH et al., 1977 , cités par LEGENDRE et LEGENDRE, $1979 ; 1983)$. De plus, les descripteurs utilisés (indices d'abondance d'espèces), s'ils sont dimensionnellement homogènes, ne sont pas tout à fait du même type, puisque le même nombre de prises par unité de capture au filet n'a pas la même signification, en fonction du potentiel de développement de chaque espèce (tel que défini par VERNEAUX, 1973) et surtout de la grégarité du taxon considéré et de sa vulnérabilité au filet maillant (RICKER, 1980) : pour une telle configuration des données, LEGENDRE et LEGENDRE (op. cit.) préconisent également le choix de l'AFC. 
Dans un premier temps, nous avons donc réalisé pour chaque campagne, une AFC globale sur les données brutes en excluant les espèces capturées à moins de 3 exemplaires par lac et en faisant figurer sur les cartes factorielles les centres d'inertie des groupements spatiaux d'une part et temporels d'autre part. Dans un deuxième temps, les variabilités spatiales et temporelles ont été décomposées en effectuant des AFC inter et intra-pôle, d'une part, puis inter et intra-séquence d'autre part. Cette approche a été réalisée en s'inspirant de la démarche développée par DOLEDEC et CHESSEL (1987 ; 1989) dans le cadre d'analyse de données tridimensionnelles, tandis que les calculs ont été effectués grâce au logiciel ADECO élaboré par ces auteurs.

\section{RÉSULTATS}

\subsection{Répartition saisonnière moyenne des captures : tendances globales}

La composition numérique des échantillons saisonniers prélevés sur les deux lacs est reportée sur le tableau III. Les deux images intersaisonnières obtenues sont dominées par le Corégone, la Perche et le Gardon, pour les deux lacs, ainsi que par le Rotengle, uniquement pour llay.

Tableau III : Composition numérique des échantillons saisonniers capturés sur llay et
sur Remoray.

Table III : Numerical composition of seasonal samples caught in the Ilay and Remoray lakes.

\begin{tabular}{|c|c|c|c|c|c|c|c|}
\hline Nom vernaculair & Nom latin & Code & $\begin{array}{c}\text { ILAY } \\
\text { PRINTEMPS }\end{array}$ & $\begin{array}{l}\text { ILAY } \\
\text { ETE }\end{array}$ & $\begin{array}{l}\text { REMORAY } \\
\text { ETE }\end{array}$ & $\begin{array}{l}\text { REMORAY } \\
\text { AUTOMNE }\end{array}$ & $\begin{array}{l}\text { PEMORAY } \\
\text { PRINTEMPS }\end{array}$ \\
\hline BROCHET & Esox lucius & BRO & $0,2 \%$ & $0,4 \%$ & $0,2 \%$ & $1,5 \%$ & $1,8 \%$ \\
\hline CARPE & Cyprinus carpio & CAR & - & - & - & $0,3 \%$ & $\cdot$ \\
\hline CHEVESNE & Leuciscus cephalus & OHE & - & - & - & $1,2 \%$ & $0,7 \%$ \\
\hline COPECONE & Coregonus sp. palea & $\infty O A$ & $36,4 \%$ & $37,0 \%$ & $19,0 \%$ & $30,9 \%$ & $33,0 \%$ \\
\hline GARDON & Rutilus rutilus & GAR & $24,8 \%$ & $29,4 \%$ & $21,3 \%$ & $46,8 \%$ & $42,5 \%$ \\
\hline GOUON & Gobio gobio & cav & - & $\cdot$ & - & $0,3 \%$ & $\cdot$ \\
\hline PERCHE & Perca fluviatilis & PER & $18,3 \%$ & $9,1 \%$ & $56,5 \%$ & $15,9 \%$ & $17,6 \%$ \\
\hline ROTENGLE & Scardinius erythrophtalmus & ROT & $14,1 \%$ & $17,4 \%$ & $0,8 \%$ & $0,9 \%$ & - \\
\hline TANCHE & Tinca tinca & TAN & $6,3 \%$ & $6,7 \%$ & $2,1 \%$ & $2,1 \%$ & $3,7 \%$ \\
\hline TRUITE DE LAC & Salmo trutta fario lacustris & TDL & - & - & - & $0,3 \%$ & $0,7 \%$ \\
\hline \multicolumn{3}{|c|}{ Effectif numérique total } & 463 & 404 & 731 & 324 & 257 \\
\hline
\end{tabular}

L'examen du tableau IV montre que les captures réalisées pour la plupart des espèces, sont réparties majoritairement, mais non exclusivement, dans un nombre de compartiments d'attraction différentielle variable selon les espèces et qui changent en fonction des saisons, ainsi que, dans une moindre mesure, d'un lac à l'autre. La variabilité importante de la fréquentation de la plupart des compartiments peut être appréciée en comparant moyenne et écart-type calculés pour les séries de captures par unité d'effort réalisées sur un même compartiment.

Ces observations sur la répartition moyenne ne font guère apparaître que des tendances globales par espèces dont il est difficile de tester la validité. En effet, l'examen des prises réalisées dans les postes affectés à un même pôle montrent des fluctuations importantes. Leur analyse nécessite l'emploi de techniques multivariées afin de déceler une éventuelle organisation spatio-temporelle des espèces les unes par rapport aux autres et autour des pôles d'attraction définis au préalable. 
Tableau IV : Répartition saisonnière des captures effectuées sur les deux lacs : moyenne et écart-type des prises numériques par unité d'effort réalisées sur chaque compartiment d'attraction différentielle.

Table IV : Seasonal distribution of catches in the two lakes : average and standard deviation of numerical catches per unit effort (CPUE) for each pole of attraction.

\begin{tabular}{|c|c|c|c|c|c|c|c|c|c|c|c|c|}
\hline \multirow{2}{*}{$\begin{array}{l}\text { ESPÈCES } \\
\text { PÓLES }\end{array}$} & \multicolumn{2}{|c|}{ BRO } & \multicolumn{2}{|c|}{ COR } & \multicolumn{2}{|c|}{ GAR } & \multicolumn{2}{|c|}{ PER } & \multicolumn{2}{|c|}{$\overline{\mathrm{ROT}}$} & \multicolumn{2}{|c|}{ TAN } \\
\hline & $m$ & $\mathbf{s}$ & $m$ & s & \multirow{2}{*}{\multicolumn{2}{|c|}{$\frac{m}{\text { ILAY }}$}} & m & $\mathbf{s}$ & m & $\mathbf{s}$ & m & 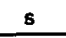 \\
\hline & & & & & & & \multicolumn{2}{|c|}{ PRINTEMPS } & & & & \\
\hline CMAX & & & 12.33 & 3,59 & 3,67 & 5,59 & 2,33 & 1,89 & 0,17 & 0,37 & & \\
\hline CMED & & & 7.83 & 2,67 & 4.67 & 6,15 & 2,50 & 3,55 & 0,50 & 0,50 & & \\
\hline CMIN & & & 2,17 & 1,46 & & & 0,50 & 0,50 & 0,17 & 0,37 & 0,17 & 0,37 \\
\hline TSUP & 0,17 & 0,37 & 1,33 & 0,74 & 1,67 & 1,70 & 3.83 & 4,81 & & & 0,50 & 0,50 \\
\hline TINF & & & & & 1,17 & 1,07 & 2,33 & 0,94 & 0,50 & 0,50 & 0,17 & 0,37 \\
\hline LFNM & & & & & 1,17 & 1,34 & 0,83 & 1,46 & 0,50 & 1,76 & 1,00 & 0,82 \\
\hline LHLD & & & & & 0,33 & 0,47 & 0,33 & 0,47 & 1,67 & 1,79 & 0,67 & 0,74 \\
\hline LHLE & & & & & 0,33 & 0,74 & 0,33 & 0,47 & 1,50 & 1,12 & & \\
\hline LHHF & & & & & 1,50 & 1,80 & & & 0,50 & 0,50 & & \\
\hline LHYF & & & & & 1,67 & 2,43 & 0,33 & 0,74 & 2,67 & 1,79 & 1.33 & 1,11 \\
\hline LBLO & & & & & 1,17 & 1,07 & 2,00 & 3,65 & 3.17 & 3,72 & 0,50 & 0,76 \\
\hline \multirow[t]{2}{*}{ par sóquence: } & 0,02 & 0,04 & 2.61 & 0,53 & 1,58 & 0,63 & 1,39 & 0,65 & 1,03 & 0,40 & 0,39 & 0,29 \\
\hline & & & \multicolumn{4}{|c|}{ ILAY } & & $\overline{E T E}$ & & & & \\
\hline CMAX & & & 8,50 & $3, \mathbf{3 0}$ & 2,50 & 2,57 & 0,17 & 0,37 & 0,50 & 0,76 & & \\
\hline CMED & & & 8,17 & 2,27 & 3,33 & 2,75 & & & 0,17 & 0,37 & 0,17 & 0,37 \\
\hline CMIN & 0,17 & 0,37 & 8.83 & 9,19 & 1,00 & 1,00 & 0,33 & 0,47 & 0,17 & 0,37 & 0,33 & 0.47 \\
\hline TSUP & & & 2,00 & 3,61 & 1,67 & 1,11 & 0,50 & 0,76 & & & & \\
\hline TINF & & & & & 1,33 & 1,11 & 1,67 & 1,19 & 0,67 & 0,74 & 0,17 & 0,37 \\
\hline LFNM & & & & & 0,33 & 0,47 & 0,83 & 1,21 & 0,50 & 0,50 & 0,67 & 0,74 \\
\hline LHLD & & & & & 0,17 & 0,37 & & & 1,50 & 1,38 & 0,33 & 0,47 \\
\hline LHLE & & & & & 1,33 & 1,25 & 0,67 & 1,11 & 1,67 & 1,19 & 0,67 & 0,47 \\
\hline LHHL & & & & & 2,50 & 3,55 & 1.88 & 2,48 & 283 & 2,97 & 0,83 & 0,69 \\
\hline LHYF & 0,17 & 0,37 & & & 3,33 & 6.15 & 0,17 & 0,37 & 2,50 & 1,49 & 1.00 & 1,00 \\
\hline LBLO & & & & & 0,69 & 0,74 & 0,33 & 0,47 & 0,17 & 0,37 & & \\
\hline par sáquence : & 0,03 & 0,05 & 2,50 & 1,13 & 1,65 & 0,92 & 0,60 & 0,35 & 0,97 & 0,45 & 0,38 & 0,19 \\
\hline & & & & & REMOF & & & ETE & & & & \\
\hline$\overline{C M A X}$ & & & 5,83 & 2,11 & 2,83 & 3,85 & 2,83 & 4,78 & & & & \\
\hline CMED & & & 10.33 & 2,92 & 2,67 & 1,60 & & & 0.33 & 0,47 & & \\
\hline CMIN & & & 8,83 & 1,57 & 3,17 & 5,30 & & & & & & \\
\hline TSUP & & & 1,17 & 0,90 & 1,67 & 1,70 & 0,50 & 0,76 & & & & \\
\hline TINF & 0,17 & 0,37 & & & 1,67 & 1,25 & 6,33 & 3,73 & & & & \\
\hline LFNM & & & & & 2,00 & 2,08 & 1,50 & 2,14 & & & 0,33 & 0,47 \\
\hline LHLD & & & & & 0,67 & 0,74 & 2,50 & 2,87 & & & 0.83 & 0,47 \\
\hline LHLE & 0,17 & 0,37 & & & 4,17 & 4,95 & 7,33 & 5,05 & & & 0,17 & 0,37 \\
\hline LHHF & & & & & 1,00 & 1,15 & 13,17 & 14,86 & & & 0,17 & 0,37 \\
\hline LHYF & & & & & 4.83 & 6,94 & 28.83 & 29,14 & 0.33 & 0,47 & 0,50 & 0,50 \\
\hline LAFF & & & & & 1,83 & 1.57 & 3,00 & 5,12 & & & 0,17 & 0,37 \\
\hline par séquence : & 0,03 & 0,06 & 2,91 & 0,50 & 2,95 & 2,04 & 7,33 & 4,20 & 0,66 & 0,09 & 0,24 & 0,11 \\
\hline & & & & & REMO & $\overline{A Y}$ & AUT & DMNE & & & & \\
\hline CMAX & & & 5,83 & 3,77 & 1,33 & 1,11 & 0,33 & 0,47 & & & & \\
\hline CMED & & & 3,67 & 1,70 & 3,83 & 4,25 & 0,17 & 0,37 & & & & \\
\hline CMIN & & & 2.50 & 4,27 & 6.33 & 6,87 & 2,00 & 2,05 & & & & \\
\hline TSUP & 0,17 & 0,37 & 0,50 & 0,76 & 3,83 & 1,21 & 3,33 & 2,06 & & & 0.33 & 0,47 \\
\hline TINF & 0,17 & 0,37 & & & 4,00 & 2,65 & 2,00 & 1,00 & & & 0,17 & 0,47 \\
\hline LFNM & & & & & 0,67 & 0,74 & 0,50 & 1,48 & & & & \\
\hline LHLD & 0,17 & 0,37 & & & 0,50 & 0,50 & & & 0.33 & 0,47 & & \\
\hline LHLE & 0,17 & 0,37 & & & 2,67 & 2,49 & 0,33 & 0,47 & & & & \\
\hline LAFF & & & & & 2,00 & 1,73 & & & & & & \\
\hline par śqquence : & 0,08 & 0,06 & 2,27 & 0,87 & 3,12 & 0,82 & 1,78 & 0,59 & 0,33 & 0,06 & 0,08 & 0,06 \\
\hline & & & & & REMC & IAY & PRINT & EMPS & & & & \\
\hline CMAX & & & 6.17 & 1,57 & 0,67 & 1,57 & 3.67 & 0,74 & & & & \\
\hline CMED & & & 4,83 & 2,41 & 2,17 & 1,95 & 1,00 & 1,15 & & & & \\
\hline CMIN & 0,17 & 0,37 & 3,33 & 2,56 & 1,67 & 2,87 & 0,34 & 0,47 & & & 0,17 & 0,37 \\
\hline TSUP & 0,17 & 0,37 & 0,33 & 0,74 & 5.50 & 2,89 & 1,00 & 1,15 & & & & \\
\hline TINF & & & 0,17 & 0,37 & 3,67 & 2,49 & 1,50 & 1,26 & & & 0.83 & 0,47 \\
\hline LFNM & & & & & 0,33 & 0,74 & & & & & & \\
\hline LHLD & 0,17 & 0,37 & & & 1,00 & 1,55 & & & & & & \\
\hline LHLE & 0,17 & 0,37 & & & 0,50 & 0,76 & & & & & 0,17 & 0,37 \\
\hline LAFF & 0,17 & 0,37 & & & 2,17 & 3,90 & 0,17 & 0,37 & & & & \\
\hline par séquence : & 0,10 & 0,05 & 1,90 & 0,28 & 2,31 & 1,16 & 1,05 & 0,02 & & & 0,14 & 0,21 \\
\hline
\end{tabular}

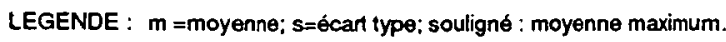




\subsection{Organisation spatio-temporelle des échantillons capturés par poste}

Les abondances numériques relatives par poste obtenues durant chaque campagne ont été soumises à l'analyse des correspondances. Les espèces ultraminoritaires ont été éliminées de l'analyse : il s'agit, pour Remoray de la Carpe, du Goujon, de la Truite de lac ainsi que du Rotengle en automne et du Chevesne au printemps ; pour le lac d'llay : le Brochet a été éliminé de l'analyse des données printanières, un seul individu ayant été capturé pendant cette campagne.

Tableau V : Pourcentage d'inertie expliquée par chacun des 3 premiers axes des AFC réalisées sur les séries saisonnières de prises par unité d'effort et pourcentage de contribution des espèces capturées à la définition de ces trois premiers axes.

Table V : Percentage of explained inertia for each of the 3 first axes of correspondence analysis carried out on the seasonal series of catches per unit effort and percentage of the contribution of the species to the definition of these three first axes.

\begin{tabular}{|c|c|c|c|c|c|c|c|c|c|c|c|c|c|c|c|}
\hline CAMPAGNES & \multicolumn{3}{|c|}{ llay Printernps } & \multicolumn{3}{|c|}{ Nlay Eté } & \multicolumn{3}{|c|}{ Remoray Eté } & \multicolumn{3}{|c|}{ Remoray Automne } & \multicolumn{3}{|c|}{ Remoray Printemps } \\
\hline AXES & $F_{1}$ & $\mathrm{~F} 2$ & F3 & F1 & F2 & F3 & F1 & $\mathrm{F} 2$ & $\overline{F 3}$ & F1 & $\mathrm{F2}$ & $\mathrm{F} 3$ & $\mathrm{~F}_{1}$ & $\mathrm{~F} 2$ & F3 \\
\hline $\begin{array}{c}\% \text { d'inertie } \\
\text { expliquée par chaque axe }\end{array}$ & 37,8 & 24,5 & 23,3 & 38,6 & 23,2 & 17,4 & 53,7 & 20,3 & 12,3 & 32,7 & 25,1 & 19,9 & 41,8 & 24,7 & 15,0 \\
\hline ESPECES & & & & bution & n\%) & différ & s esp & ésa la & finitior & chact & $\operatorname{tes} 3$ & miers & & & \\
\hline$\overline{B R O}$ & - & - & - & 0,0 & 0,2 & 0.4 & 0,1 & $\overline{0.6}$ & 89,6 & 9.9 & 0.1 & 82.4 & 1,8 & 2.6 & 65.6 \\
\hline $\mathrm{CHE}$ & - & - & - & - & - & - & - & - & - & 0,5 & 0,1 & 3,2 & - & - & - \\
\hline $\mathrm{COA}$ & 32,3 & 12,1 & 0,1 & 49,1 & 2,2 & 6,6 & 70,1 & 7.8 & 0.0 & 54,3 & 7,9 & 2,7 & 46,1 & 12,0 & 0,0 \\
\hline GAR & 0,3 & 8,1 & 34,7 & 1,0 & 15,6 & 49,4 & 0,2 & 69,5 & 0.0 & 4,8 & 38,3 & 2,4 & 44,0 & 5.6 & 8,9 \\
\hline PER & 0,1 & 3,6 & 64,5 & 14,3 & 38,2 & 37.5 & 29.1 & 16.2 & 0.0 & 28,4 & 50.7 & 4,3 & 2,0 & 73,0 & 0,1 \\
\hline ROT & 61,8 & 21,4 & 0,6 & 25,6 & 19,2 & 1,1 & 0,0 & 0,0 & 0,3 & - & - & - & - & - & - \\
\hline TAN & 5,6 & 54,7 & 0,2 & 10,0 & 24,6 & 5.0 & 0.6 & 5,9 & 10.1 & 2,1 & 3,0 & 5,1 & 3,5 & 3,0 & 25,5 \\
\hline TRU & - & - & - & - & - & - & - & - & - & - & - & - & 2,6 & 3,7 & 0,0 \\
\hline
\end{tabular}

Pour chaque campagne, le calcul dégage deux axes nets qui totalisent un pourcentage d'inertie expliquée allant de $58 \%$ à $74 \%$ suivant les campagnes (cf. tableau V). Quelle que soit l'analyse considérée, ce sont les espèces ultra-majoritaires (Corégone, Gardon, Perche ainsi que Rotengle, uniquement pour llay) qui assurent la quasi-totalité de la contribution aux deux premiers axes (cf. tableau $V$ ) et non les espèces rares. Sur ce tableau, on constate également que, pour les trois campagnes réalisées à Remoray, la définition de l'axe 3 est assurée essentiellement par le Brochet. Les ordinations obtenues pour les cinq analyses étant du même type, et afin de ne pas alourdir l'exposé, seules les cartes factorielles correspondant à la campagne estivale effectuée sur Remoray sont présentées sur les figures 4 et 5 , les plans F1F2 obtenus pour les autres campagnes étant reproduits en annexe. La suite de l'analyse confirmera d'ailleurs que les variabilités spatiotemporelles se décomposent de la même façon pour les 5 jeux de données (cf. figure 6).

En outre, compte tenu du fort pourcentage d'inertie expliquée par les deux premiers axes, les plans F1F2 peuvent être privilégiés pour analyser les phénomènes étudiés. Afin de visualiser la variabilité des captures par pôle (dans l'espace) et par échantillon global ou séquence (dans le temps), la projection des postes prospectés sur le plan défini par les deux premiers axes (plan F1F2) est représenté en double sur la figure 4. La fenêtre logique figurant sur le coin supérieur droit des cartes factorielles indique le rapport hauteur/largeur réel de la projection du nuage de points qui a été légèrement modifié par le changement d'échelle ("rescaling") opéré pour des raisons de mise en page.

Sur la partie haute de cette représentation graphique, les points correspondant aux 6 postes affectés à un même pôle sont reliés par des segments de droite à leur centre d'inertie qui localise le pôle d'attraction correspondant dans le plan de l'analyse. Les espèces sont également situées sur cette carte factorielle. Le degré de dispersion des postes autour de leur centre d'inertie figurant le pôle d'attraction est variable : il est en général important pour les pôles littoraux, moyen ou mineur pour les pôles sublittoraux et centraux. Par ailleurs, ces nuages de 6 points, même s'ils sont intersécants sur le plan F1F2, occupent des secteurs différents de l'espace abstrait; en particulier les 3 ensembles 
de pôles correspondant respectivement aux zones centrale, sublittorale et littorale sont nettement séparés. Cependant, d'un lac à l'autre, et même d'une campagne à l'autre, la position des pôles d'attraction varie ainsi que leur écart relatif.

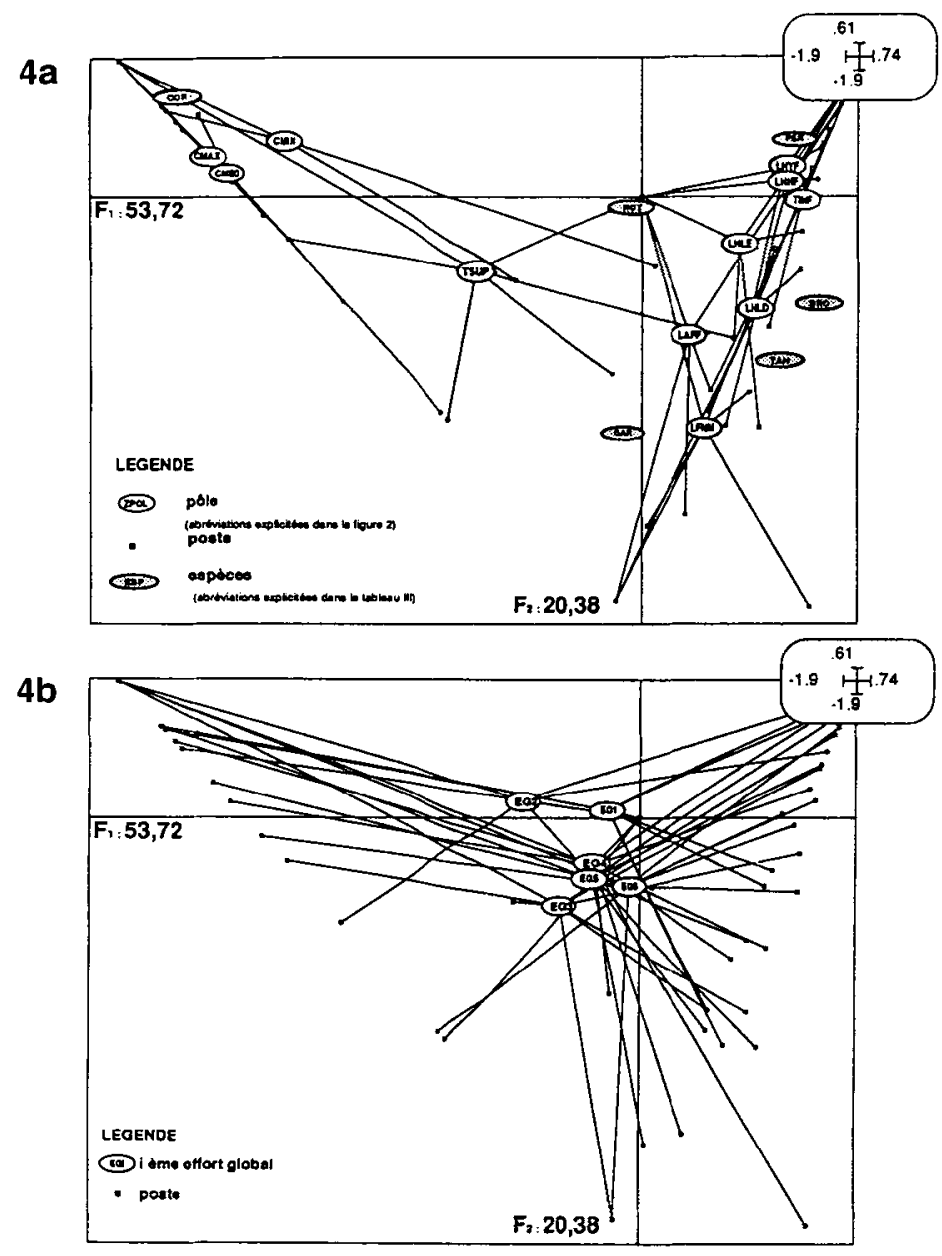

Figure 4 : Distribution des postes groupés par pôles (4a) et par efforts globaux (4b) sur le plan des 2 premiers axes factoriels de l'AFC réalisée sur les prises par unités d'effort effectuées dans le lac de Remoray en été ; la carte factorielle 4a représente également la distribution des espèces.

4a : Les 6 postes prospectés pour chacun des 11 pôles sont reliés à leur centre d'inertie qui figure ce pôle.

4b : Les 11 postes (1 par pôle) prospectés simultanément pendant chacun des 6 efforts globaux sont reliés à leur centre d'inertie qui représente cet effort global.

Figure 4 : Distribution of fishing posts grouped by pole (4a) and by global effort (4b) on the plan of the first two factorial axes of the correspondence analysis carried out on the catches per unit effort during the summer in Remoray ; the factorial map 4a also represents the distribution of species on the first plan of the analysis.

4a : The six fishing posts prospected for each pole are related to their inertia center which represents this pole.

4b : The eleven posts prospected simultaneously during each global effort are related to their inertia center which represents this global effort. 
Sur la partie basse de la figure 4, la même carte factorielle est représentée, mais ce sont les points correspondant aux 11 postes prospectés pendant un même effort global d'échantillonnage qui sont reliés à leur centre d'inertie. Celui-ci localise donc la position, sur le plan F1F2, de l'image du peuplement obtenue par chacune des 6 prospections unitaires de l'ensemble des pôles. Le bon degré de coïncidence des structures des 6 nuages de 11 points figurant les 6 efforts globaux successifs et la proximité des 6 centres d'inertie correspondant aux différentes combinaisons spatiales de la communauté étudiée durant une campagne montre que les efforts.globaux successifs donnent des images de l'activité du peuplement similaires ou au moins cohérentes entre elles.

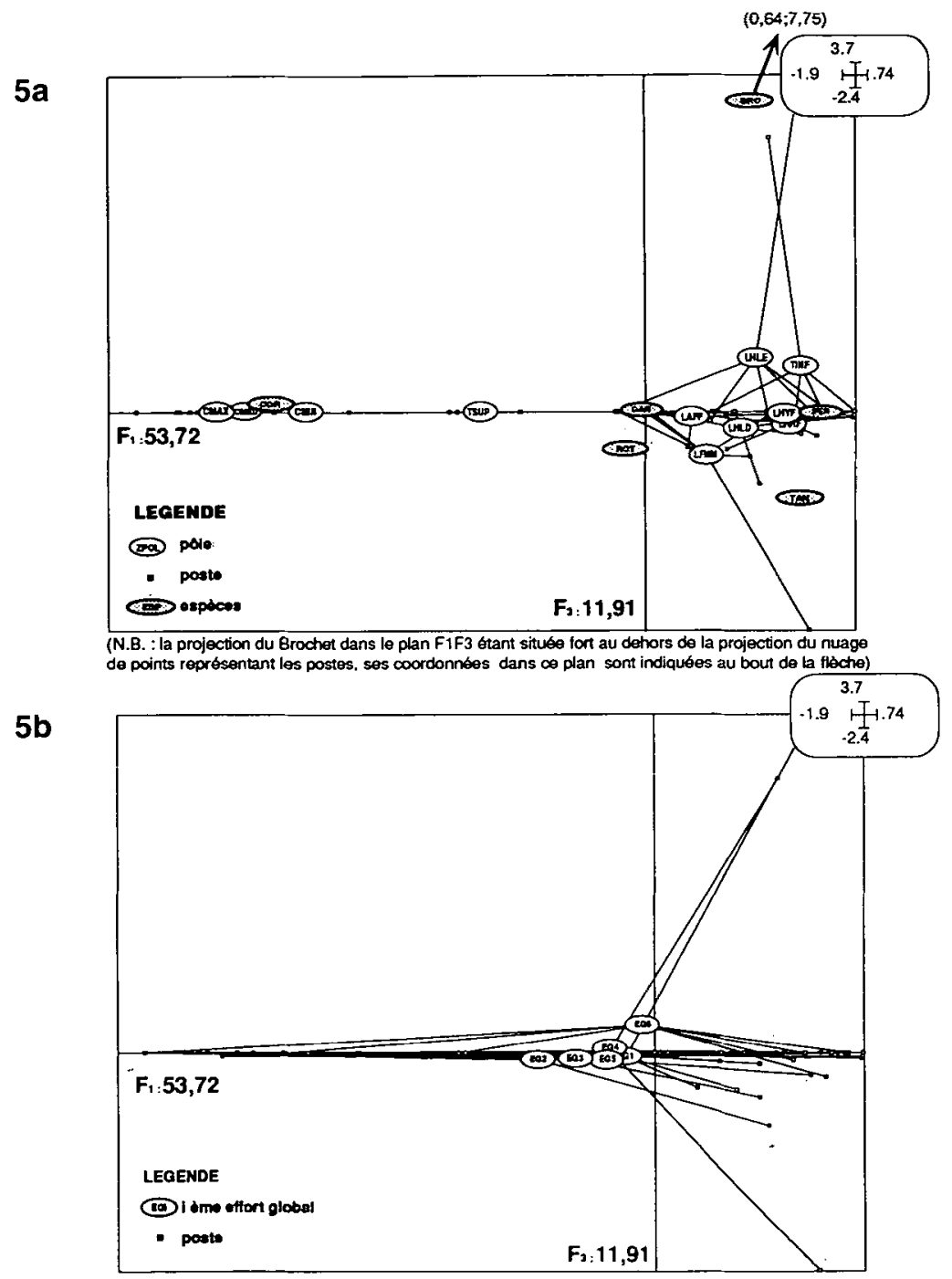

Figure 5 : Distribution des postes groupés par pôles (5a) et par efforts globaux (5b) sur le plan F1F3 de l'AFC réalisée sur les prises par unités d'effort effectuées dans le lac de Remoray en été ; les cartes factorielles $5 a$ et $5 b$ sont organisées comme les deux cartes de la figure 4.

Figure 5 : Distribution of fishing posts grouped by pole (5a) and by global effort (5b) on the plan F1F3 of the correspondence analysis carried out on the catches per unit effort during the summer in Remoray ; the factorial maps $5 a$ and $5 b$ are organized like the two maps of the figure 4.

Le plan $\mathrm{F} 1 \mathrm{~F} 3$, également représenté en double suivant la même dualité de regroupement sur la figure 5 pour la campagne estivale de Remoray, montre une dispersion moindre des pôles mais révèle la même proximité des six séquences entre elles. 


\subsection{Décomposition de la variabilité totale suivant les effets spatiaux et temporels}

Les AFC inter et intra-pôles, ainsi que inter et intra-séquences, réalisées en suivant la démarche établie par DOLEDEC et CHESSEL $(1987,1989)$, dégagent à chaque fois un premier axe à forte inertie expliquée $(32,7 \%$ à $86,7 \%)$. La première valeur propre de chacune de ces analyses, prenant en compte la variabilité due à l'effet étudié (DOLEDEC et CHESSEL, op. cit. ), est reportée sur la figure 6 qui permet de les comparer.

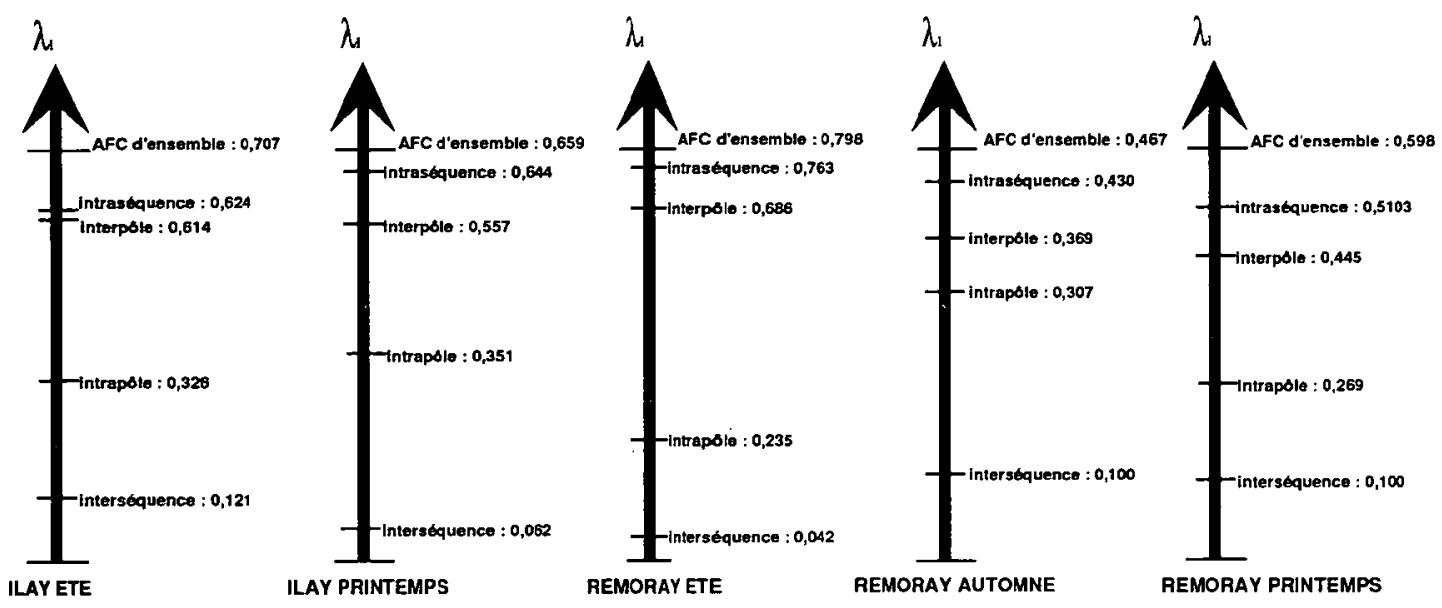

Figure 6 : Comparaison des premières valeurs propres obtenues en réalisant 2 AFC intergroupes et 2 AFC intragroupes sur chaque jeu saisonnier de données groupées par pôles (effet spatial) et par séquence (effet temporel) ; chacune de ces premières valeurs propres prend en compte la variabilité induite par l'effet correspondant (DOLEDEC et CHESSEL,1987,1989).

Figure 6 : Comparison of the first eigen values obtained by the calculation of two intergroups then two intragroups correspondence analysis on each seasonal series of data grouped by pole (spatial effect) and by global effort (temporal effect); each one of these eigen values takes into consideration the variability due to the corresponding effect (DOLEDEC et CHESSEL, 1987, 1989).

Quels que soient la campagne et le lac considérés, les variabilités attribuées aux différents effets spatiaux et temporels se classent de la même manière : la plus grande partie de la variabilité totale serait due, d'abord à la différence entre les postes prospectés au même moment (au cours d'une même séquence), puis à la disparité globale des pôles les uns par rapport aux autres, en terme de tendance moyenne. La variabilité induite par les différences de captures par unité d'effort entre les différents postes rattachés à un même pôle, si elle s'avère notable, reste à chaque fois nettement inférieure à la variabilité calculée d'un pôle à l'autre. De façon plus accentuée encore, la variabilité calculée d'une séquence à l'autre est minime, et ce pour toutes les campagnes.

\section{DISCUSSION - CONCLUSIONS}

\subsection{Nature de l'organisation spatiale des espèces capturées}

La proportion importante d'inertie expliquée par les deux premiers axes des analyses factorielies globales montre l'existence d'une organisation logique nette entre les espèces et les postes prospectés. Sur les cartes factorielles correspondantes, les captures par unité d'effort sont ordonnées selon des gradients marqués, jalonnés par les pôles d'attraction.

L'analyse de la contribution des différents postes et espèces du plan F1F2, des chiffres bruts des captures réalisées, ainsi que la confrontation des regroupements spatiotemporels opérés, permettent d'avancer des hypothèses prudentes quant à la signification 
des deux premiers axes. L'ordination des postes suivant le premier axe de chaque AFC globale par campagne pourrait correspondre au gradient de complexité croissante de la topographie aquatique induisant une variation conjointe du complexe paramétrique physico-chimique local et donc déterminant de façon dynamique les arrangements spatiaux de la communauté. Le deuxième axe pourrait exprimer, quant à lui, les variations des taux de fréquentations, à la fois quantitative (abondance des captures) et qualitative (variété des captures), associés aux postes.

Si la position relative des pôles et leurs écarts relatifs varient à travers les systèmes et surtout les saisons, on retrouve des tendances stables quant à l'utilisation des pôles, en particulier d'un lac à l'autre tandis que sur l'ensemble des cartes factorielles, les 3 zones restent typiquement individualisées. D'ailleurs, la variabilité globale au sein des pôles, appréhendée par le calcul d'une AFC intra-pôle, est nettement inférieure à la variabilité d'un pôle à l'autre déterminée par AFC inter-pôle (cf. figure 6).

Ces différentes observations sont compatibles avec l'hypothèse de l'attractivité différentielle des compartiments de l'espace lacustre pré-défini et soulignent les variations qualitatives et quantitatives de cette attractivité suivant les systèmes et les saisons.

\subsection{Aspects dynamiques des combinaisons spatiales mises en évidence}

Au sein d'une même campagne, la dispersion des postes autour des pôles, plus ou moins marquée selon la zone considérée, traduit l'utilisation variable des compartiments définis au cours d'une même saison. L'ensemble de la communauté pourrait s'organiser selon le modèle décrit pour le Brochet par CHAPMAN et MCKAY, 1984 : chaque individu (ou banc selon les espèces) se déplace à travers le systèmes en exploitant, durant une période de "sédentarité" d'une durée déterminée, un ou plusieurs compartiments de l'espace lacustre. Cette "versatilité" ou, selon les point de vue, cet "opportunisme" est attribué, à des degrés divers, à l'ensemble des espèces vivant en eau profonde par de nombreux auteurs (LARKIN, 1956 ; MOREAU et LEGENDRE, 1979 ; SAVARD et MOREAU, 1982).

Cependant, chaque combinaison spatiale peut être considérée comme étant structurée de façon dynamique par l'attractivité différentielle des compartiments définis sur le plan lacustre, puisque les différents pôles prospectés au même moment montrent une disparité notoire (cf. figure 6), alors que les séries de 6 prospections simultanées de l'ensemble des pôles fournissent une image similaire ( $\mathrm{cf}$. partie basse des figures 4 et 5) et réduisent la variabilité au minimum (cf. figure 6).

\subsection{Variation de l'organisation spatiale de l'ichtyofaune d'un lac à l'autre}

Si l'attractivité des différents pôles varie d'un lac à l'autre, le type d'organisation générale des captures par unité d'effort autour de ces pôles reste le même pour les lacs d'llay et de Remoray, distants de $70 \mathrm{~km}$ et bien différenciés selon leurs caractéristique morphologiques respectives. Cependant, ces deux lacs, situés dans la même zone biogéographique, présentent, en outre, certaines similitudes physico-chimiques : on peut donc se demander si le modèle proposé et vérifié pour ces deux cas restera valable pour des systèmes plus nettement différents. Une approche identique menée sur un lac plus grand (420 ha, $65 \mathrm{~m}$ de profondeur maximum) constitué par une retenue artificielle située en contexte géologique siliceux, la retenue du Vieux Pré (Meurthe-et-Moselle, France), a d'ores et déjà, fourni des résultats similaires et mis en évidence une organisation identique des captures autour de pôles d'attraction définis de la même manière (MNEP, 1992).

Par conséquent, cette étude montre l'intérêt, souligné par DOLEDEC et CHESSEL (1991), d'incorporer les objectifs dans l'analyse. L'utilisation de résultats préalables nous a, en effet, permis de réaliser un découpage a priori du plan lacustre et d'élaborer une stratégie d'échantillonnage précise, suivant un type d'approche également recommandée par FRONTIER (1983) et SHERRER (1983). La répétition de l'échantillonnage systématique et simultané de l'ensemble des compartiments pré-définis nous a alors permis de mettre en évidence le caractère dynamique des arrangements spatiaux réalisés par les poissons puis de mesurer et d'interpréter la variabilité du modèle proposé. 
Ce découpage du plan lacustre constitue, d'après les critères indiqués par SHERRER (op. cit.) et FRONTIER (op. cit.), une stratification acceptable de l'échantillonnage, puisque d'une part la variabilité intra-strate est inférieure à la variabilité inter-strate, et que, d'autre part, la répétition de l'échantillonnage global montre une faible variabilité des résultats globaux. Ce dernier point se traduit, pour chaque espèce, par la possibilité d'estimer et d'améliorer, en répétant l'effort global, la précision des mesures d'abondance "'relative" que constituent les captures par unité d'effort de pêche au filet.

Cette approche de la nature et du type de l'organisation spatiale des poissons permet donc d'envisager l'élaboration d'un protocole d'échantillonnage reproductible d'un système lacustre à l'autre. Ce standard, dont la précision pourra être estimée, devrait permettre des comparaisons intersystémiques sur l'état ou l'évolution de l'ichtyofaune ainsi que des expérimentations sur la Biologie et l'Ecologie des espèces.

Annexe 1 : Distribution des postes groupés par pôles (la) et par efforts globaux (lb) sur le plan F1F2 de l'AFC réalisée sur les prises par unités d'effort effectuées dans le lac d'llay au printemps; les cartes factorielles la et lb sont organisées comme les deux cartes de la figure 4.

Appendix I : Distribution of fishing posts grouped by pole (la) and by global effort (lb) on the plan F1F2 of the correspondence analysis carried out on the catches per unit effort during springtime in llay; the factorial maps la and $\mathrm{lb}$ are organized like the two maps of the figure 4.

la

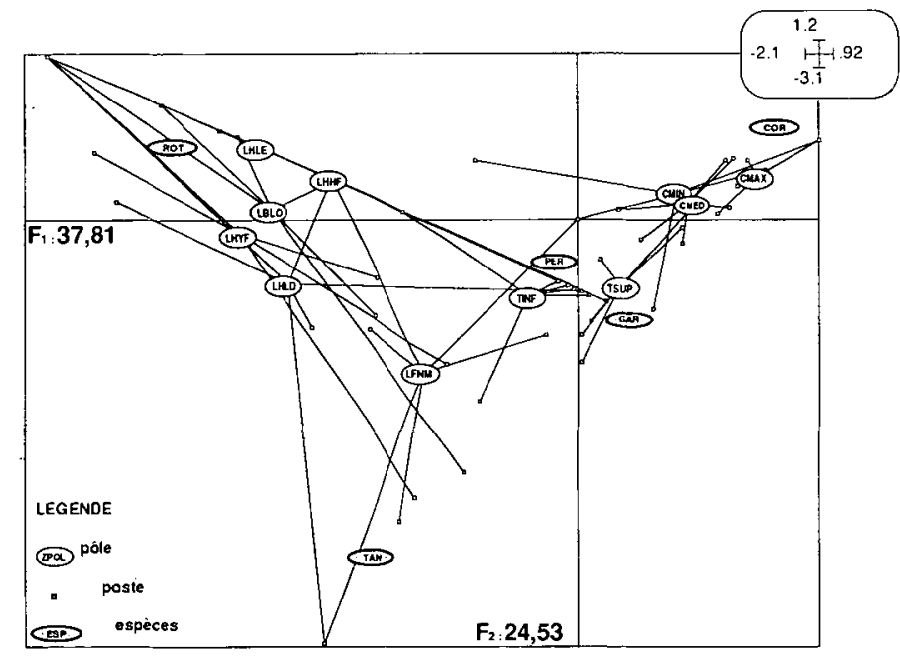

Ib

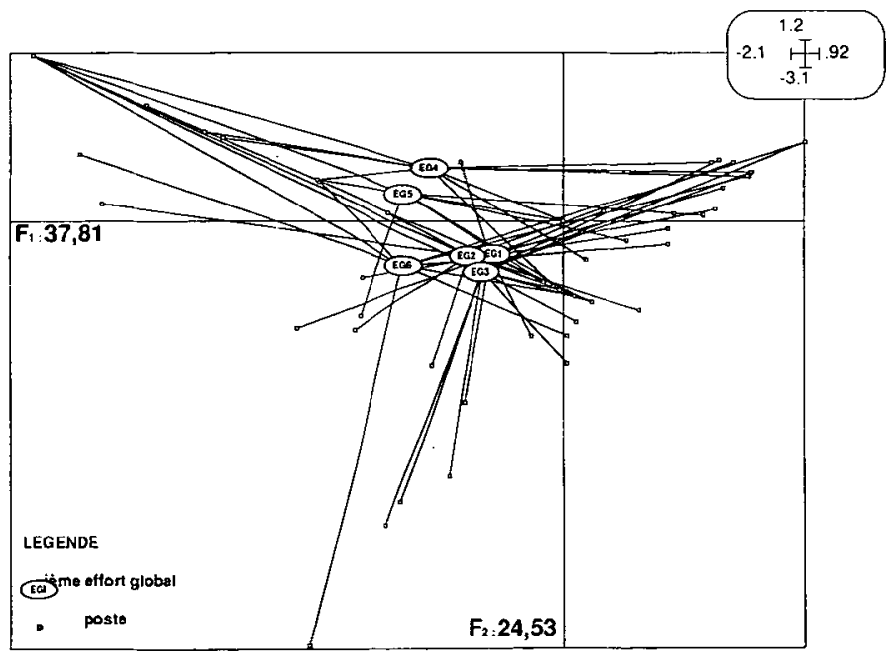


Annexe II : Distribution des postes groupés par pôles (Ila) et par efforts globaux (IIb) sur le plan F1F2 de l'AFC réalisée sur les prises par unités d'effort effectuées dans le lac d'llay en été ; les cartes factorielles lla et Ilb sont organisées comme les deux cartes de la figure 4.

Appendix II : Distribution of fishing posts grouped by pole (Ila) and by global effort (IIb) on the plan F1F2 of the correspondence analysis carried out on the catches per unit effort during the summer in llay; the factorial maps Ila and Ilb are organized like the two maps of the figure 4.
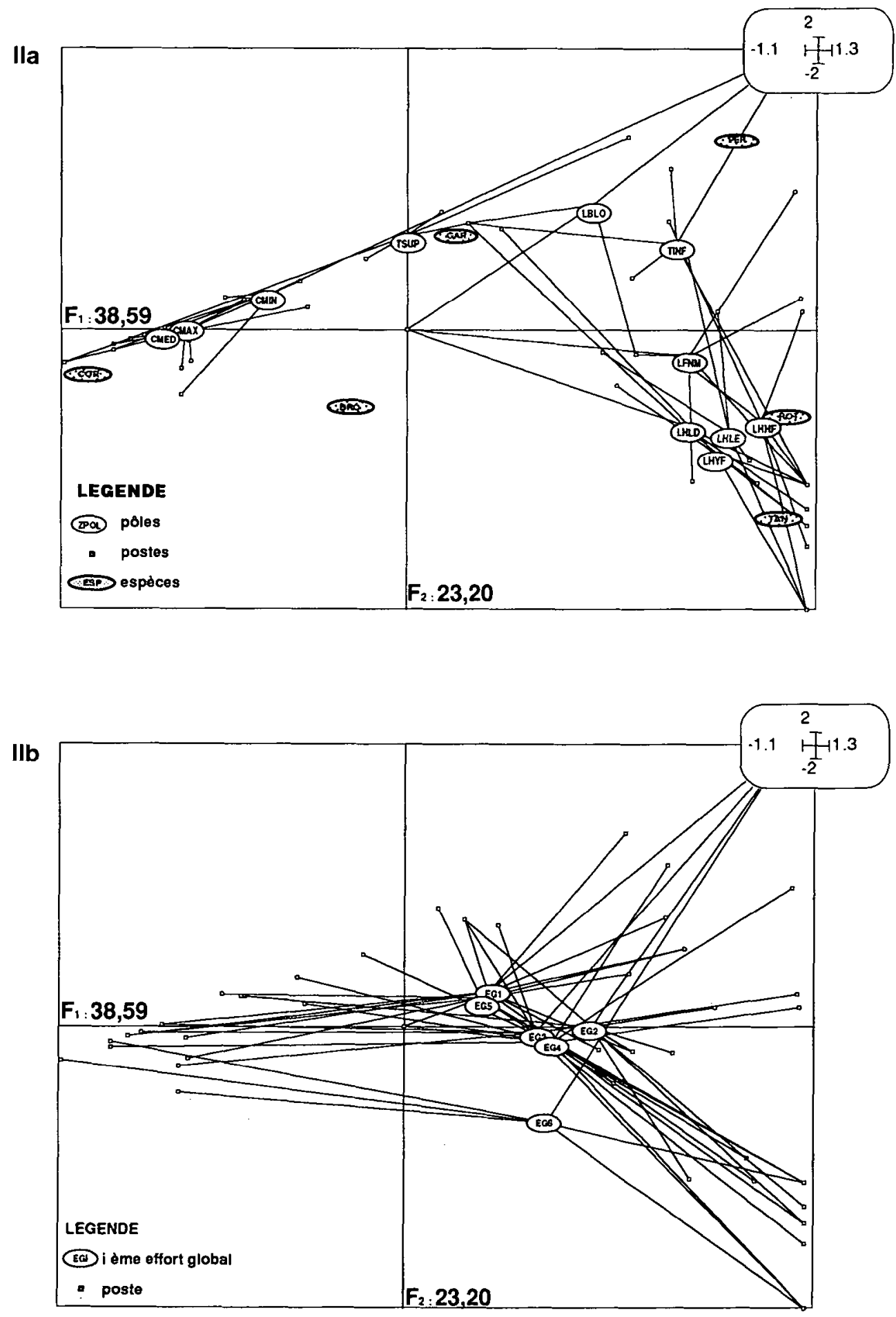
Annexe III : Distribution des postes groupés par pôles (IIla) et par efforts globaux (IIIb) sur le plan F1F2 de l'AFC réalisée sur les prises par unités d'effort effectuées dans le lac de Remoray en automne; les cartes factorielles Illa et IIIb sont organisées comme les deux cartes de la figure 4.

Appendix III : Distribution of fishing posts grouped by pole (IIla) and by global effort (IIIb) on the plan F1F2 of the correspondence analysis carried out on the catches per unit effort during the autumn in Remoray ; the factorial maps IIla and IIIb are organized like the two maps of the figure 4.
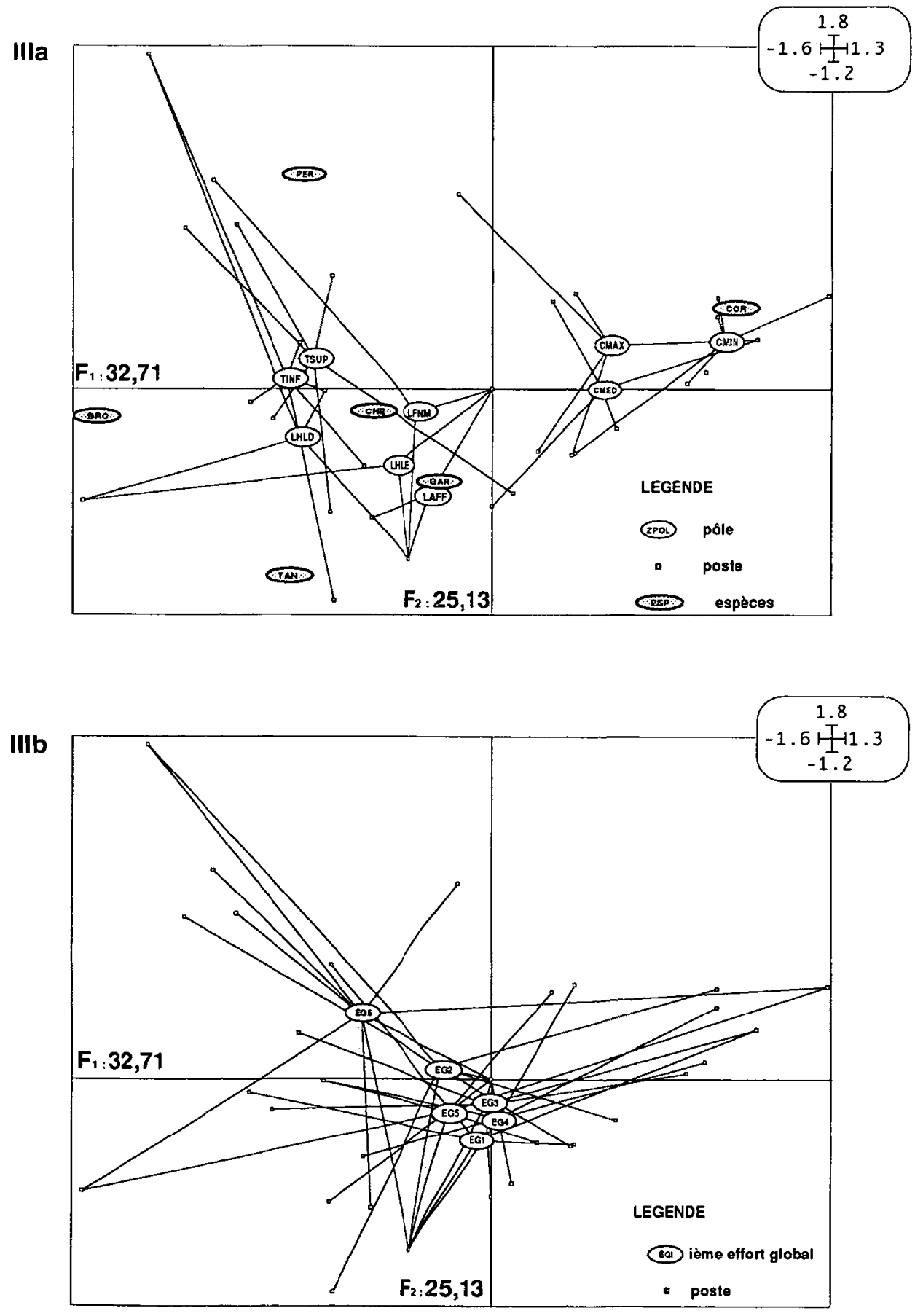
Annexe IV : Distribution des postes groupés par pôles (IVa) et par efforts globaux (IVb) sur le plan F1F2 de l'AFC réalisée sur les prises par unités d'effort effectuées dans le lac de Remoray au printemps ; les cartes factorielles IVa et IVb sont organisées comme les deux cartes de la figure 4 .

Appendix IV : Distribution of fishing posts grouped by pole (IVa) and by global effort (IVb) on the plan F1F2 of the correspondence analysis carried out on the catches per unit effort during the springtime in Remoray; the factorial maps IVa and IVb are organized like the two maps of the figure 4.

IVa

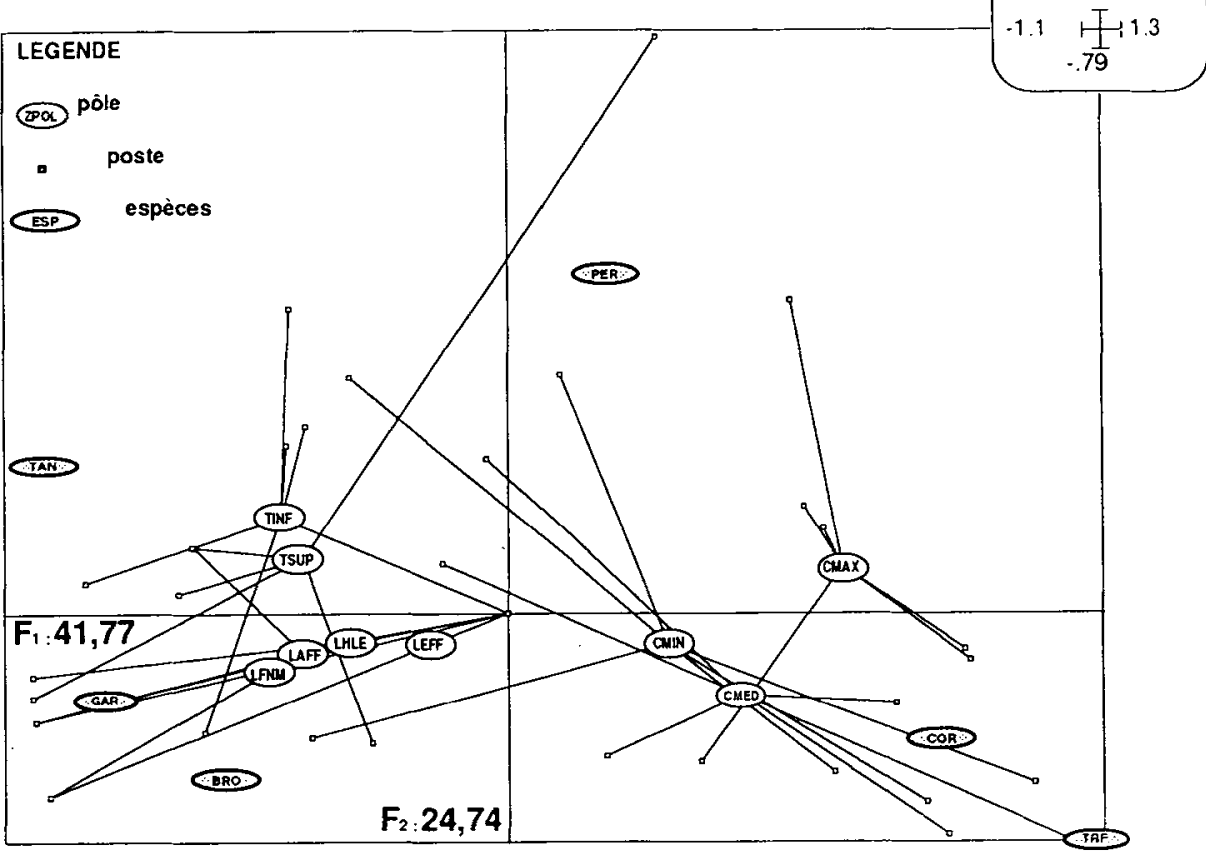

IVb

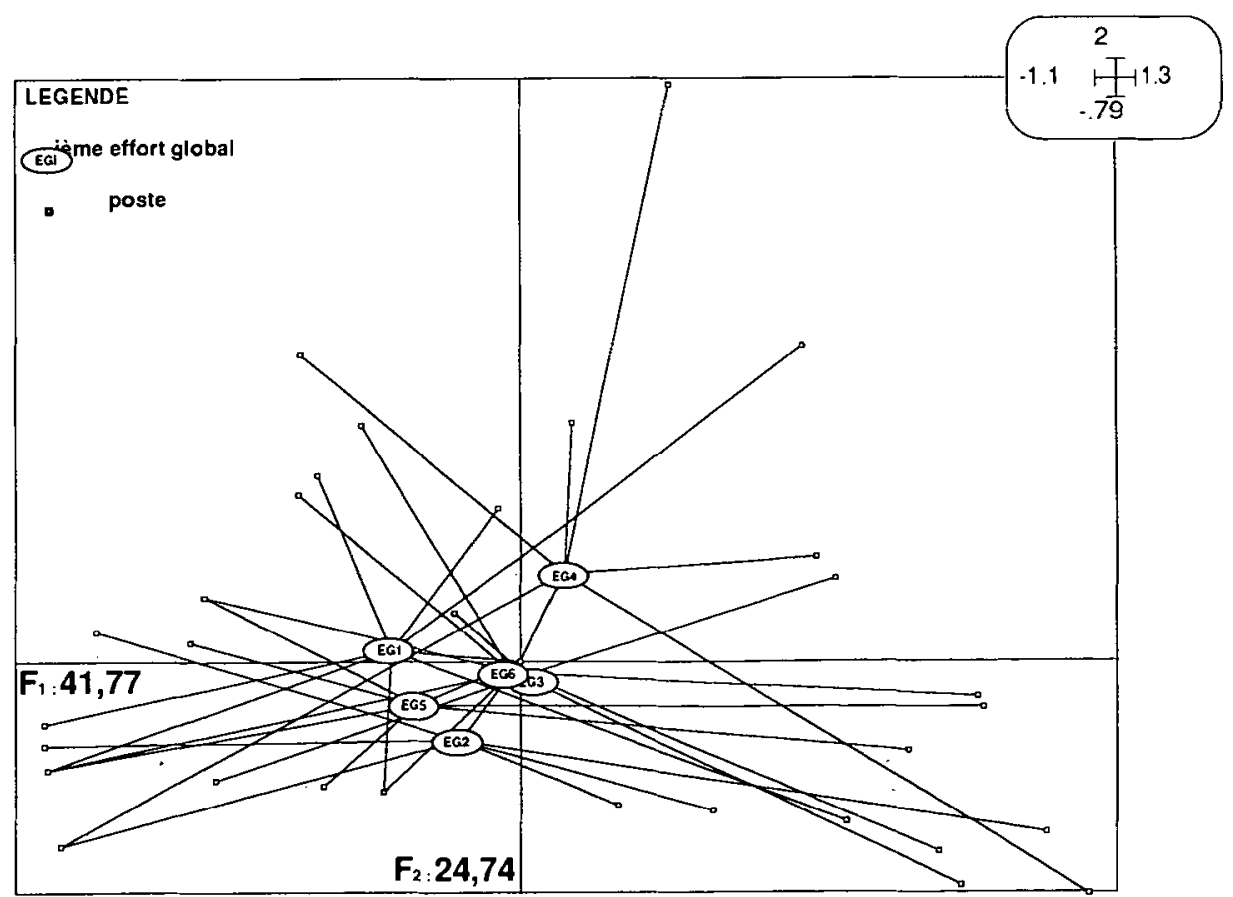




\section{REMERCIEMENTS}

Les auteurs tiennent à remercier chaleureusement :

- Fabiola LANCHIPA, pour son aide lors de la traduction en anglais du résumé et des légendes.

- André ROUSSELET, garde commissionné du CSP (détaché auprès de la Fédération Départementale de Pêche du Doubs) dont les remarques, fruit d'une longue expérience de la pêche au filet, nous ont fort aidé à mettre au point la technique proposée et à préciser les pôles d'attraction.

- les Fédérations Départementales de Pêche du Jura et du Doubs, ainsi que la Maison de la Réserve de Remoray, pour nous avoir accordé les droits de pêche et d'accès indispensables.

- MM. les Professeurs GUYARD et VERNEAUX (ISTE de Besançon) pour leurs conseils, remarques et critiques constructives qui nous ont aiguillés et aiguillonnés tout au long de notre travail.

\section{BIBLIOGRAPHIE}

BARLA M.J., 1991. Species composition, richness and diversity of fish assemblages in different habitats of a pampean lake (Argentina). Annls Limnol., 27(2), 163-173.

BARTOO N.W., HANSEN R.G., WYDOVSKI R.S., 1973. A portable vertical gillnet system. Prog. Fish. Cultur., 35, 231-233.

BENZECRI J.P. \& Coll., 1973. L'analyse des données. II-L'analyse des correspondances. Bordas, Paris, $620 \mathrm{p}$.

BOVEE K.D., 1978. The incremental method of assessing habitat potential for cool water species with management implications. Trans. Amer. Fish. Soc. spec. publ., 11, 340346.

CEEC (Centre d'Etude des Eaux Continentales de Besançon), 1988. L'habitat lacustre et les peuplements pisciaires. Ministère de l'Environnement, rapport S.R.E.T.I.E. $n^{\circ} 87079,52 \mathrm{p}$.

CHAPMAN C.A., MCKAY W.C.,1984. Versatility in habitat use by a top predator, Esox lucius (L.). J. Fish. Biol., 25, 109-115.

CROWDER L.B., COOPER W.E., 1982. Habitat structural complexity and the interaction between the bluegills and their prey. Ecology, 63, 1802-1803.

DAKKI M., 1985. Sur le choix des données en biotypologie des eaux courantes par l'analyse factorielle des correspondances. Bull. Ecol., 16(4), 285-296.

DEGIORGI F., GRANDMOTTET J.P., 1992. Impact de la désoxygénation chronique d'un lac de moyenne montagne sur son ichtyofaune. Ichtyophysiologica Acta, 15, 79-97.

DIANA J.S., MCKAY W.C., EHRMANN M., 1977. Movement and habitat preferences of northern Pike, Esox lucius (L.), in lake Ste Anne, Alberta. Trans. Amer. Fish. Soc. 106(6), 561-565.

DIEHL S., 1988. Foraging efficiency of three freshwater fishes : effect of structural complexity and light. Oikos, 53, 1802-1813.

DIONNE M., FOLT C.L., 1991. An experimental analysis of macrophytes growth forms as fish foraging habitat. Can. J. Fish. Aquat. Sci., 48, 123-131.

DOLEDEC S., CHESSEL D., 1987. Rythmes saisonniers et composantes stationnelles : I Description d' un plan d'observation complet par projection de variables. Acta CEcologica CEcol. Gener.,8(3), 403-406.

DOLEDEC S., CHESSEL D., 1989. Rythmes saisonniers et composantes stationnelles en milieu aquatique II - Prise en compte et élimination d'effets dans un tableau faunistique. Acta CEcologica CEcol. Gener., 10(3), 207-232. 
DOLEDEC S., CHESSEL D., 1991. Recent development in linear ordination methods for environmental sciences in Trends in Ecology, Council of Scientific Research Integration Ed., Research Trends (Publishers),(sous presse).

DUSSART B., 1966. Limnologie : l'étude des eaux continentales. Gauthier Villars Ed. , Paris, $674 \mathrm{p}$.

FRONTIER S., 1983. Choix et contrainte de l'échantillonnage en écologie in Frontier S., Stratégie de l'échantillonnage en écologie, 15-62, Masson Ed., Paris.

GASCON D., LEGGET W., 1977. Distribution abundance and resource utilization of littoral zone fishes in response to a nutrient / production gradient in lake Memphremagog. $J$. Fish. Res. Board Can., 34, 1105-1117.

GAUCH H.G., WHITTAKER R.H., WENTWORTH T.R., 1977. A comparison study of reciprocal averaging and other techniques. J. Ecol., 65, 157-174.

GORMANN O.T., KARR J.R., 1978. Habitat structure and stream fish communities. Ecology, 59(3), 507-515.

GRANDMOTTET J.P., 1983. Principales exigences de 30 téléostéens dulcicoles vis-à-vis de l'habitat aquatique.Ann. Sci. Univ. Fr. Comté, Biol. anim., 4(4) , 3-32.

GRANDMOTTET J.P., VAUDAUX P., 1989. Utilisation des filets verticaux pour l'échantillonnage des peuplements pisciaires : premiers résultats et perspectives. Actes du colloque de l'IGGE, Juin $89,17 p$.

GUYARD A., GRANDMOTTET J.P., VERNEAUX J., 1989. Utilisation de batteries de filets verticaux à enroulement : nouvelle technique d'échantillonnage de la faune ichtyologique lacustre. Application à l'étude du peuplement pisciaire de la retenue du barrage de Vouglans (Jura). Ann. Sci. Univ. Fr. Comté, Biol. anim., 5(1), 59-70.

HARALDSTAD O., JONSSON B., 1983. Age and sexe segregation in habitat utilization by brown trout in a Norvegian Lake. Trans. Am. Fish. Soc., 112, 27-37.

HARTMANN G.F., 1962. Use of gill net rollers in fisheries investigations. Trans. Am. Fish. Soc., 91, 224-225.

HILL M.O.,1973. Reciprocal averaging : an eigenvector method of ordination.J. Ecol., 61, 237-249.

HINDAR K., JONSSON B., 1982. Habitat and food segregation of dwarf and normal arcticcharr (S. alpinus) from Vangsvanet lake. Western Norway. Can. J. Fish. Aquat. Sc., $39,1030-1045$.

HORARK D.L., TANNER H.A., 1964. The use of vertical gill nets in studying fish depth distribution. Trans. Am. Fish. Soc., 103(2) , 348 -352.

HUET M.,1949. Aperçu des relations entre la pente et les populations piscicoles dans les eaux courantes. Schweiz Z. Hydrol., 11(3-4), 332-351.

HUTCHINSON J., 1957. A treatrise on Limnology. Vol. 1. J. Wiley \& Son inc., New York, $1001 \mathrm{p}$.

ILLIES J., BOTOSANEANU L., 1963. Problèmes et méthodes de la classification et de la zonation écologiques des eaux courantes, considérées surtout du point de vue faunistique. Mitt. internat. Verein Limnol., 12, 1-57.

LARKIN P. A., 1956. Interspecific competition and population control in fresh water. J. Fish. Res. Board Can., 13, 327-342.

LEGENDRE P., BEAUVAIS A., 1978. Niches et association de poissons des lacs de la Radissonie québécoise. Naturalist. can., 105, 137-158.

LEGENDRE L., LEGENDRE P., 1979. Ecologie numérique. Tome II : la structure des données écologiques. Masson Ed., Paris, $254 \mathrm{p}$.

LEGENDRE L., LEGENDRE P., 1983. Echantillonnage et traitements des données. In Frontier S., Stratégie de l'échantillonnage en écologie, 163-215, Masson Ed., Paris. 
LINDSTRÖM T., BERGSTRAND E. , 1979. The habitat of perch, Perca fluviatilis L., on the outskirts of its swedish distribution, lakes and lakes reservoirs. Inst. Freshwat. Res., 58, 55-76.

MNEP, 1992. Etude piscicole de la retenue du Vieux Pré. Rapp. EDF, 81 p.

MOREAU G., LEGENDRE L., 1979. Relation entre habitat et peuplement de poisson : essai de définition d'une méthode numérique pour des rivières nordiques. Hydrobiologia, 67, 81-87.

RICKER W.E., 1980. Calcul et interprétation des statistiques biologiques des populations de poissons. Bull. Can. Sci. Lal. aq., Ottawa, 409 p.

SAVARD L., MOREAU G., 1982. Etude des relations entre les communautés piscicoles et les différents habitats d'une rivière nordique; notion d'habitat optimal. Can. J. of Zool., $60,3344-3352$.

SHERRER B., 1983. Technique de sondage en écologie. In Frontier S., Stratégie de l'échantillonnage en écologie, 63-162, Masson Ed., Paris.

TONN W.M., MAGNUSON J.J., 1982. Patterns in the species composition and richness of fish assemblages in northern Wisconsin lakes . Ecology 63(4), 1149-1166.

VERNEAUX J., 1973. Cours d'eau de Franche-Comté (Massif du Jura). Recherches écologiques sur le réseau hydrographique du Doubs. Thèse d'Etat. Univ. Fr. Comté, Besançon, $257 \mathrm{p}$.

VERNEAUX J., VIDONNE A., REMY F., GUYARD A., 1991. Particules organiques et rapport $\mathrm{C} / \mathrm{N}$ des sédiments des lacs du Jura. Annis Limnol., 27(2), 175-190. 